Barbara Schneider

Neuwied, Deutschland

\title{
Die Behandlung des chronischen Rückenschmerzes - die Rolle der TCM im tagesklinischen Setting
}

Dass die Behandlung des chronischen Rückenschmerzes multimodal erfolgen sollte, ist mittlerweile Konsens, ebenso das Anliegen, in erster Linie aktive Verfahren einzusetzen. Welchen Beitrag die TCM vor diesem Hintergrund leisten kann, werde ich anhand unserer Erfahrungen aus der Arbeit in unserer Schmerztagesklinik darstellen.

\section{Akupunktur - mehr als Technik} Schmerzpatienten sind in der Regel durch viele Praxen und Diagnostik gegangen. Sie haben gleichermaßen viel Durchführung von Techniken an ihrem Körper erlebt. So reiht sich die Akupunktur zunächst recht unauffällig in diese Kette ein.

Nach unterschiedlichen Konzepten werden Akupunkturrezepte abgeleitet und durchgeführt. Gemäß dem Gedanken Ben und Biao geschieht dies individuell. Damit lassen sich in den allermeisten Fällen positive Veränderungen, im Sinne von Entspannung und Schmerzreduktion, herbeiführen.

Akupunktur kann jedoch weit mehr liefern als Technik. Während der Durchführung tritt der Akupunkteur zwangsläufig in Kontakt mit dem Patienten. Dieser Kontakt ist ausgestaltbar und intensivierbar, durch Nachfragen und Anleiten zum Spüren, ganz zentral im Rahmen der Palpation Based Acupuncture. Es entsteht Kommunikation.

Neben dem Nadeln der Punkte kann der Patient zur Akupressur angeleitet werden. Dies bietet ihm die Möglichkeit, selbst aktiv zu sein und entsprechend Selbstwirksamkeit zu erfahren. Er kann damit, wenn nötig unter Anleitung, in Beziehung zum eigenen Körper treten.

Deutsche Zeitschrift für Akupunktur

2018 • 61 (3): 156

https://doi.org/10.1007/s42212-018-0082-3

Online publiziert: 3. August 2018

(c) Springer Medizin Verlag GmbH, ein Teil von

Springer Nature 2018

\section{Qi als alternatives Pathogenese- modell}

Mit ihrem energetischen Ansatz bietet die TCM ein alternatives Pathogenesemodell für den Schmerz. Das kann kreativ eingesetzt werden.

Indem man z. B. der Diagnose „degeneratives LWS-Syndrom" das stagnierte Qi als gedankliche Alternative anbietet, kann es gelingen, den Patienten aus seinem angestrengten Suchen nach der einen entscheidenden Ursache herauszuhelfen. So tritt die Funktion in den Fokus und weniger die Struktur; erstgenannte ist veränderbar! Statt „entweder oder“ kann dann „sowohl als auch" gedacht werden.

Wenn es gelingt, den Rückenschmerz als , in seinem Fluss gestörtes Qi“ zu verstehen, bieten sich viele gedankliche und daraus abgeleitete Verhaltensmöglichkeiten.

Mögliche Übersetzungen für die Aufgabe: „sorge dafür, dass dein Qi gut fließ $\mathrm{t}$ “, könnten z. B. sein, „bewege dich mehr“, „ändere häufiger deine Körperhaltung", „wärme, klopfe, reibe“. Es kann aber auch die Aufforderung sein, dafür zu sorgen, ausreichend Qi zur Verfügung zu haben. Dies kann heißen, sich wertvoller und regelmäßiger zu ernähren, häufiger in der Natur aufzuhalten, Augen, Ohren und Nase zu öffnen oder ein gutes Verhältnis von Pause und Aktivität zu finden.

Vor dem Hintergrund der Lebenspflege können Qigong, Yoga oder Tai-Chi als wirksame Techniken eingeführt werden.

\section{In Bildern denken - der Link zur Psychologie}

Das Modell der 5 Wandlungsphasen mit ihrer Vielzahl an Entsprechungen stellt eine Schatzkiste dar. Auf sie kann gerade, wenn es um Emotionen rund um den Schmerz geht, gut zugegriffen werden. Viele unserer Patienten sind sehr sensibel in Bezug auf psychologische Schmerztherapie und fühlen sich schnell in die sog. Psychoecke gedrängt. Durch Analogien kann es gelingen, über Dinge zu spre- chen, ohne sie direkt auszusprechen. Der Patient behält die Kontrolle darüber, was thematisiert wird und inwieweit er übertragen möchte.

Die aufgekommenen Themen stehen gleichrangig neben Themen wie Beweglichkeit, Muskelspannung oder Schlaf.

\section{Fazit}

Das System der TCM bietet einen hervorragenden Rahmen für multimodales Vorgehen vor dem Hintergrund eines biopsychosozialen Entstehungsmodells chronischer Schmerzen.

Wir führen 4-wöchige teilstationäre Programme mit je 6 Patienten durch. Je nach Patientengruppe nutzen wir die oben geschilderten Ansätze als Arbeitsgrundlage unterschiedlich intensiv. Auch im Hinblick auf die weitere Betreuung der Patienten und längerfristige Verhaltensänderungen trägt der gewählte Ansatz.

Die zentralen Begriffe der multimodalen Schmerztherapie und der TCM, wie Bewegung, Autonomie, Selbstwirksamkeit, Akzeptanz oder Ressourcen, teilen sich eine inzwischen riesige Schnittmenge. Dies sollte man nutzen!

\section{Korrespondenzadresse}

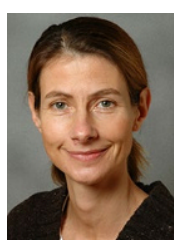

Dr. med. Barbara

Schneider

Friedrich-Ebert-Str. 36,

56564 Neuwied,

Deutschland

barbara@schneider-amlangdiek.de

Einhaltung ethischer Richtlinien Interessenkonflikt. B. Schneider gibt an, dass kein Interessenkonflikt besteht.

Dieser Beitrag beinhaltet keine von den Autoren durchgeführten Studien an Menschen oder Tieren. 